\title{
Video-assisted left main bronchial carcinoma resection and secondary carinal reconstruction
}

\author{
Rong Yin, MD, PhD, ${ }^{\mathrm{a}}$ Ninglei Qiu, MD, ${ }^{\mathrm{a}}$ Jun Zhu, MD, ${ }^{\mathrm{b}}$ and Lin $\mathrm{Xu}, \mathrm{MD}, \mathrm{PhD},{ }^{\mathrm{a}}$ Nanjing, China
}

Video-assisted thoracoscopic surgery (VATS) is becoming increasingly popular in the field of general thoracic surgery; however, VATS sleeve lobectomy still remains a big challenge for the thoracic surgeon. ${ }^{1}$ The left secondary carinal reconstruction, a more complicated bronchoplasty procedure, has rarely been reported even in with open thoracic surgery. Here we present the case of a left main bronchus carcinoma partially invading the left upper bronchus. This patient underwent partial left main bronchus and upper bronchus resection with pulmonary preservation and a secondary carinal reconstruction in a complete VATS procedure. This case report was approved by our hospital's ethics committee.

\section{CLINICAL SUMMARY}

A 61-year-old man was admitted to our hospital for a left main bronchial mass with symptoms of productive cough and blood-stained sputum for longer than 2 months. A chest computed tomographic scan and bronchoscopy revealed a $15-\mathrm{mm}$ endobronchial mass obstructing roughly $80 \%$ of the left main bronchial lumen (Figure 1, $A$ and $B$ ). Histologic examination of an endobronchial biopsy specimen confirmed squamous cell cancer. The patient had a history of left renal clear cell carcinoma 3 years previously, for which he had undergone a radical nephrectomy. Preoperative positron emission tomography, computed tomographic examination, and brain magnetic resonance imaging did not find any evidences of recurrence of renal clear cell carcinoma or metastasis of the left main bronchial tumor either. This patient's lung function was poor because of a 60 packyear history of smoking. The forced expiratory volume in 1 second and maximum voluntary ventilation were only $61.1 \%$ and $52.2 \%$ of predicted values, respectively. Arterial blood gas analysis showed that $\mathrm{PaO}_{2}$ and $\mathrm{PaCO}_{2}$ were 64.9 and $42.7 \mathrm{~mm} \mathrm{Hg}$, respectively.

\footnotetext{
From the Department of Thoracic Surgery, ${ }^{\mathrm{a}}$ Nanjing Medical University Affiliated Cancer Hospital, Cancer Institute of Jiangsu Province, Nanjing, China; and the Department of Radiotherapy, ${ }^{\mathrm{b}}$ Nanjing Medical University Affiliated Cancer Hospital, Cancer Institute of Jiangsu Province, Nanjing, China.

Disclosures: Authors have nothing to disclose with regard to commercial support.

Received for publication Dec 11, 2012; revisions received Jan 22, 2013; accepted for publication Feb 13, 2013; available ahead of print March 13, 2013.

Address for reprints: Lin Xu, MD, PhD, Department of Thoracic Surgery, Nanjing Medical University Affiliated Cancer Hospital, Cancer Institute of Jiangsu Province, 42 Baiziting, Nanjing, 210009, PR China (E-mail: xulin_83@yahoo.cn).

J Thorac Cardiovasc Surg 2013;145:e60-2

0022-5223/ $\$ 36.00$

Copyright (c) 2013 by The American Association for Thoracic Surgery

http://dx.doi.org/10.1016/j.jtcvs.2013.02.038
}

The operation was conducted completely as VATS, with a $30-\mathrm{mm}$ anterolateral utility incision placed in the left fourth intercostal space, and another pair of 12-mm ports placed in the seventh intercostal space on the posterior axillary line and the sixth intercostal space on the midaxillary line, 1 for the videoscope and the other for the thoracoscopic instruments (Figure 2, A). No rib spreading was done.

The first important step was isolating pulmonary artery trunk and removing lymph nodes nearby bronchi, which was helpful to release the tension on the subsequent anastomosis. Then the partial left main bronchus and upper bronchus with the luminal tumor was cut free of the bronchi with a scalpel under the bronchoscopic guidance (Figure 2, B). After negative margins were ascertained with frozen sectioning, the reconstruction of the left secondary carina was conducted. First, the membranous part of the left upper and lower bronchi were sutured together with 4 interrupted 4-0 absorbable sutures (Vicryl; Ethicon, Inc, Somerville, NJ), and the knots were tied on the outside with a knot pusher (Figure 2, $C, F$, and $G$ ). After this, the anastomosis between left main bronchus and the combination of upper and lower bronchi was performed with a continuous 3-0 Vicryl suture (Figure 2, D-G). A Crile nerve hook was used to pull the suture tight after each stitch. Then the bronchoscope was used again to verify the intact of anastomosis, without kinking or retained secretions. Finally, a systematic lymph node dissection was performed.

There were no perioperative complications. Chest tubes were removed on postoperative day 5 , and the patient was discharged in good condition on day 10 . The final pathology report showed a $15-\times 10-\mathrm{mm}$ polypoid intraluminal grade II squamous cell cancer, and focal regional invasion of the lamina propria was observed. All the dissected lymph nodes and margins were negative. The patient received $40 \mathrm{~Gy}$ adjuvant postoperative radiotherapy 6 weeks after resection. The patient recovered well, without any complications and evidences of relapse at 8-month follow-up. The thoracic computed tomographic scan at 8 months after the operation (Figure 1, C-E) showed the left anastomotic stoma to be unobstructed, and no pulmonary atelectasis was observed.

\section{DISCUSSION}

To our knowledge, this is the first report describing a secondary carinal reconstruction through a complete VATS 

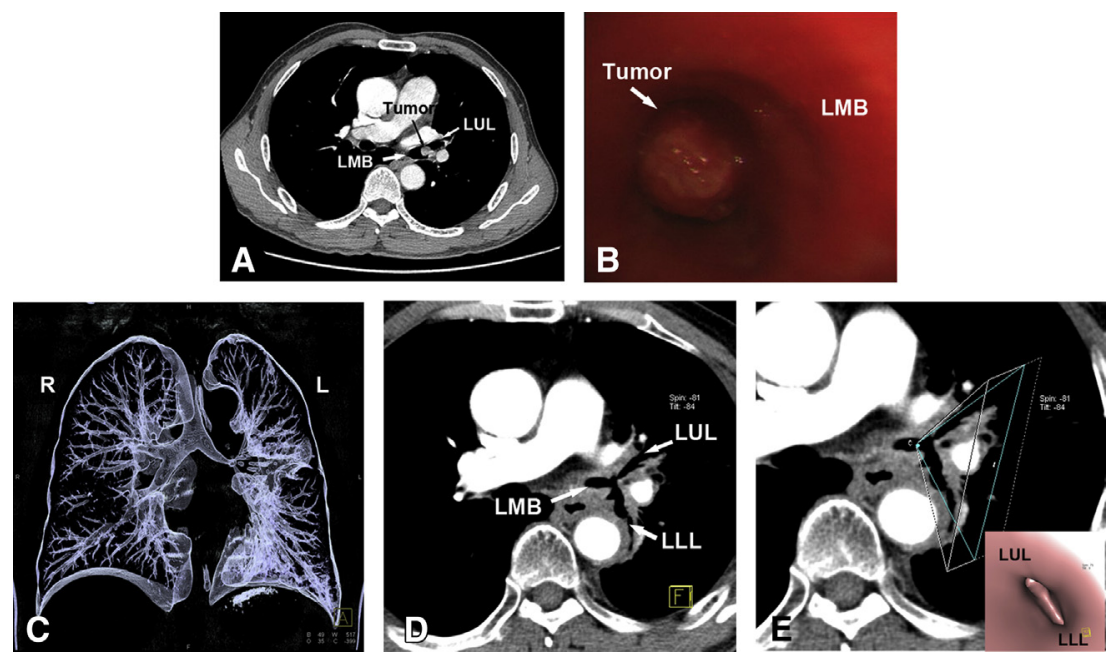

FIGURE 1. Representative preoperative images from computed tomographic scan (A) and bronchoscopic examination (B). The arrow shows an endobronchial tumor obstructing roughly $80 \%$ of the left main bronchial lumen. C, The bronchial tree with 3-dimensional reconstruction shows that no pulmonary atelectasis was observed. Postoperative images from computed tomographic scan (D) and 3-dimensional reconstruction (E) at 8 months after operation. The anastomotic stoma was unobstructed, and no pulmonary atelectasis was observed. $L U L$, Left upper lobe bronchus; $L M B$, left main bronchus; $L L L$, left lower lobe bronchus.

approach. The tumor was located at the left main bronchus and closed to the orifice of upper bronchus. Obviously, an upper sleeve lobectomy is much safer to guarantee a negative margin; however, concerns regarding poor lung function meant that even a lobectomy might significantly decrease quality of life. We therefore compromised and treated the patient as for a tracheal tumor by performing a secondary carinal reconstruction. Because this was an

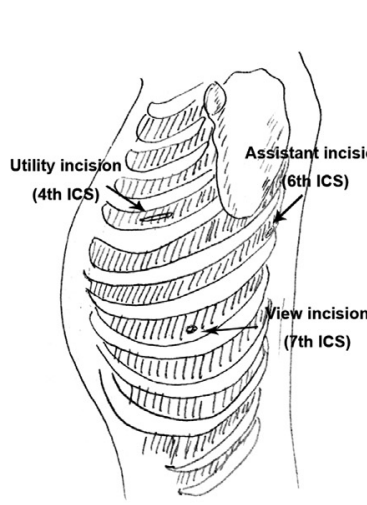

A

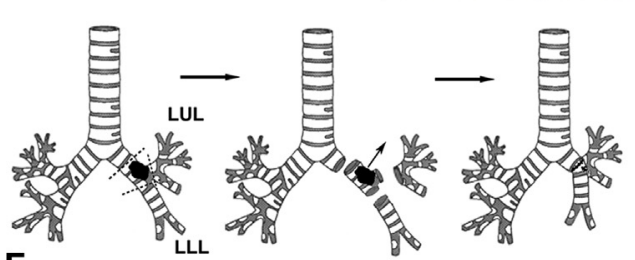

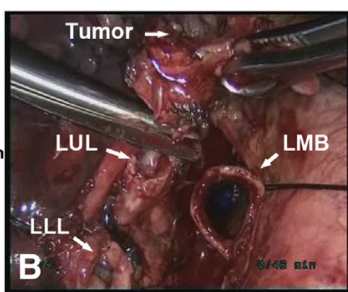
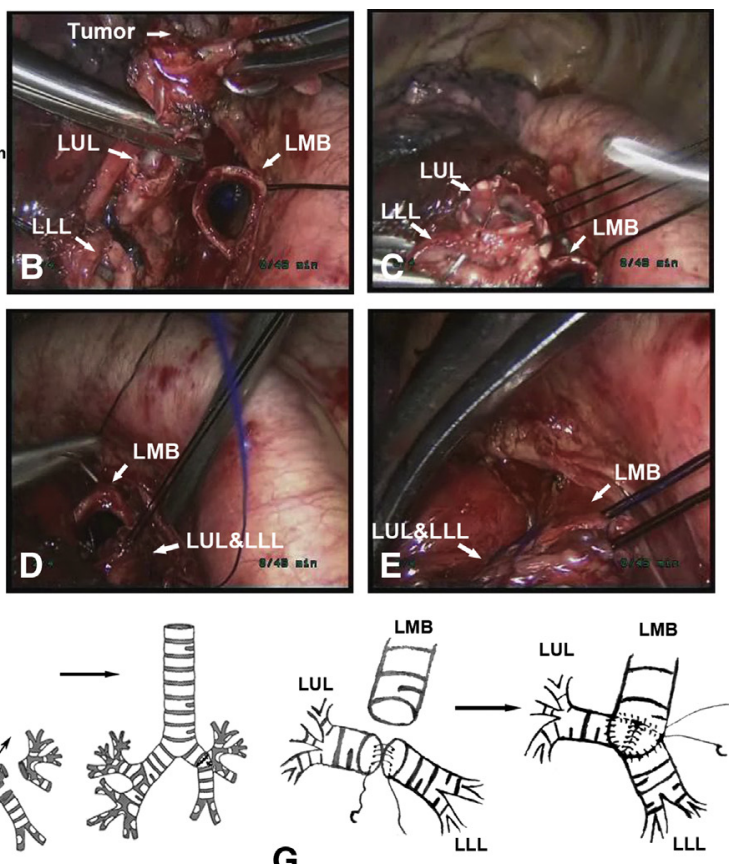

G

FIGURE 2. A, A schematic diagram illustrates the positioning of the 3 incisions. Representative photos of left main bronchus ( $L M B$ ) tumor resection and left secondary carinal reconstruction: the luminal tumor is cut free of the bronchus (B), interrupted sutures are placed in the membranous part of left upper lobe bronchus $(L U L)$ and the left lower lobe bronchus $(L L L ; \mathrm{C})$; and the anastomosis is completed between the left main bronchus and the combination of left upper and lower lobe bronchi (D and E). Schematic diagrams of operative procedures (F) and secondary carinal reconstruction (G). ICS, Intercostal space. 
intraluminal tumor, bronchoscopy and frozen sections allowed us to guarantee a negative margin and still preserve lung function. Obviously, it was a big challenge to conduct the anastomosis of 3 tubes, including the left main bronchus, left upper bronchus, and left lower bronchus, in a complete VATS procedure. Such a task is best attempted by surgeons experienced in both advanced VATS and bronchoplasty to deal with such variant suture angles and long anastomotic length. Because the advantage of VATS lobectomy versus open surgery has been well established, ${ }^{2}$ however, we think that our operation will hold better functional benefits for this patient.

Finally, according to the experience of MD Anderson Cancer Center, ${ }^{3}$ adjuvant postoperative radiotherapy can improve the disease-specific survival for patients with tracheal or bronchial squamous cell cancer. Although there is no consensus on the role of adjuvant radiotherapy, we therefore believe that postoperative radiotherapy was also very important for this patient.

\section{References}

1. Mahtabifard A, Fuller CB, McKenna RJ Jr. Video-assisted thoracic surgery sleeve lobectomy: a case series. Ann Thorac Surg. 2008;85:S729-32.

2. McKenna RJ Jr, Houck W, Fuller CB. Video-assisted thoracic surgery lobectomy: experience with 1,100 cases. Ann Thorac Surg. 2006;81:421-5; discussion 425-6.

3. Webb BD, Walsh GL, Roberts DB, Sturgis EM. Primary tracheal malignant neoplasms: the University of Texas MD Anderson Cancer Center experience. J Am Coll Surg. 2006;202:237-46.

\section{Unique case of total artificial cardiac support in failed Fontan circulation after cardiectomy: Is continuous flow better than pulsatile flow?}

Christina J. VanderPluym, MD, Nee S. Khoo, MD, Ivan M. Rebeyka, MD, and Holger Buchholz, MD, Edmonton, Alberta, Canada

We report a case of total cardiectomy with implantation of a left ventricular assist device (VAD) for postcardiotomy Fontan circulation failure. This unique experience provides valuable insights into the physiologic consequences of pulsatile versus continuous flow (CF) VADs in the singleventricle circulation.

\section{CLINICAL SUMMARY}

A male infant with tricuspid atresia 1C underwent pulmonary artery banding shortly after birth, followed by a bidirectional cavopulmonary anastomosis at 6 months. At 2.8 years of age, he underwent an extracardiac fenestrated Fontan procedure and was extubated in the operating room. Excessive chest tube losses prompted reexploration 8 hours after surgery. Uncontrollable hemorrhage from an

From the Division of Cardiology and Cardiovascular Surgery, Department of Pediatrics, Stollery Children's Hospital, University of Alberta, Edmonton, Alberta, Canada.

Disclosures: Authors have nothing to disclose with regard to commercial support.

Received for publication Dec 14, 2012; revisions received Feb 6, 2013; accepted for publication Feb 13, 2013; available ahead of print March 20, 2013.

Address for reprints: Christina J. VanderPluym, MD, Division of Pediatric Cardiology, University of Alberta \& Stollery Childrens Hospitals, Edmonton, Alberta,

Canada (E-mail: christina.vanderpluym@ childrens.harvard.edu).

J Thorac Cardiovasc Surg 2013;145:e62-3

$0022-5223 / \$ 36.00$

Copyright (c) 2013 by The American Association for Thoracic Surgery

http://dx.doi.org/10.1016/j.jtcvs.2013.02.031 extensive myocardial hematoma was identified. The patient was placed on cardiopulmonary bypass; however, further measures to control bleeding from a myocardial dehiscence along the diaphragmatic surface of the heart were unsuccessful. The heart was completely excised along the atrioventricular groove while maintaining the Fontan pathway intact, and the ascending aorta was transected at the sinotubular junction with removal of all native valves. A 30-mm Gelweave graft (Terumo Cardiovascular Systems Corporation, Ann Arbor, Mich) was anastomosed to the left atrial cuff, forming a reservoir for pulmonary venous return and connected to a 9-mm Berlin Heart (BH) inflow cannula. A 16-mm Gore-Tex graft (W. L. Gore \& Associates, Flagstaff, Ariz) was sewn end-to-end to the ascending aorta, permitting placement of a 6-mm BH outflow cannula. A CentriMag CF pump (Thoratec Corporation, Pleasanton, Calif) was connected to the $\mathrm{BH}$ cannulas as an interim device during the wait for patient stabilization. The CentriMag resulted in stable flow, with a mean arterial pressure of 50 to $70 \mathrm{~mm} \mathrm{Hg}$ and a central venous pressure of 12 to $14 \mathrm{~mm} \mathrm{Hg}$. An $80-\mathrm{mL}$ saline tissue expander was placed within the empty pericardial sac to maintain space for future transplantation.

The patient was extubated, alert, and interactive on day 6 of support. At day 10, a mobile thrombus in the circuit necessitated pump change. In light of the patient's hemodynamic stability and improving end organ function, he the 